\title{
Un modèle alternatif et innovant de Recherche et Développement pour garantir l'accès aux médicaments
}

\section{Éditorial \\ Maladies négligées, populations négligées}

et système d'innovation biomédicale

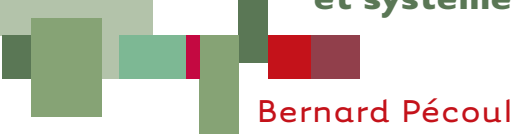

> Le prix exorbitant des médicaments fait la une des médias depuis plusieurs mois. Rien qu'en France, de nombreuses initiatives ont vu le jour cette année : 110 cancérologues ont lancé un appel afin de dénoncer l'explosion des prix des nouveaux anti-cancéreux, Médecins du Monde (MdM) a rappelé à l'ordre la ministre des Affaires sociales et de la Santé sur le rôle fondamental de l'État à réguler les coûts de la santé, prenant pour exemple l'hépatite $C$ dont le médicament le plus efficace, le Sovaldi ${ }^{\circledR}$, coûte plus de 40000 euros par patient et est le symptôme même d'un système qui dysfonctionne.

Si la situation est critique en France concernant le prix de nouveaux médicaments, aux États-Unis, ce même débat a été évoqué lors de la campagne présidentielle et d'importantes controverses ont vu le jour au sujet de médicaments développés il y a des décennies, dont les prix ne cessent d'augmenter. Le cas le plus récent est celui de l'EpiPen ${ }^{\circledR}$, un antihistaminique dont le prix a augmenté de $550 \%$ en quelques années. On se souvient en 2015 du scandale «Martin Shkreli $»^{1}$, ce dernier ayant décidé d'augmenter le prix du médicament Daraprim ${ }^{\circledR}$, utilisé notamment contre la toxoplasmose, de 13,50 \$ à 750 \$ en une nuit.

La question du prix des médicaments et de l'accès se pose partout dans le monde et pèse sur l'équilibre des comptes de la Sécurité sociale pour la France. Plus globalement, la question posée est la suivante : «y-a-t-il un lien entre le prix des médicaments et les coûts investis dans la R\&D ? » Ces questions sont maintenant débattues au sein des plus grandes instances internationales, comme le montre la publication en septembre dernier du rapport du Groupe de haut niveau des Nations-Unies pour l'accès aux médicaments?'

\section{Un nouveau modèle de Recherche et Développement}

Ces préoccupations étaient déjà d'actualité au début des années 2000 , lorsqu'un nouveau modèle de R\&D est né : les partenariats de développement de produits (PDP), dont fait partie l'initiative Médicaments contre les Maladies Négligées (DNDi, Drugs for Neglected Diseases initiative). Ce qui a motivé la création de DNDi, en 2003, a été le cruel constat des docteurs de Médecins Sans Frontières (MSF), quotidiennement confrontés à l'absence de traitements efficaces et à un prix abordable pour un grand nombre de patients atteints de maladies négligées. L'absence de marché lucratif constitué par ces

${ }^{1}$ Martin Shkreli, le patron d'un fond d'investissement hedge fund, a augmenté le prix du médicament après avoir racheté le laboratoire qui le produisait.

${ }^{2}$ Voir le rapport complet : https://staticl.squarespace.com/static/562094dee4b0d00cla3ef761/ t/57d9c6ebf5e231b2f02cd3d4/1473890031320/UNSG+HLP+Report+FINAL+12+Sept+2016.pdf patients détournait la recherche médicale de l'urgent besoin de développer des traitements adaptés pour ces populations dites « négligées ». DNDi a donc été formée par des institutions de recherche médicale dans le monde, en particulier dans les régions les plus endémiques, et avec le soutien de MSF, de l'Institut Pasteur et de l'Organisation mondiale de la santé (OMS), afin de créer un «laboratoire virtuel » de recherche avec des partenariats publics et privés, modèle qui limite au maximum les coûts de la R\&D et qui accélère la mise à disposition d'un nouveau traitement.

Les maladies tropicales négligées ont été le premier cœur de cible de ce modèle alternatif de R\&D. Maladie du sommeil ${ }^{3}$, leishmaniose, maladie de Chagas $^{4}$, paludisme, autant de maladies parasitaires, pour certaines peu connues, qui touchent plus d'un milliard de personnes dans le monde. Afin de rapidement répondre aux besoins urgents des patients, DNDi et ses partenaires ont mis en place une stratégie de recherche à court-terme, visant une amélioration immédiate des options thérapeutiques existantes, notamment avec des combinaisons ou des associations médicamenteuses, ou des nouvelles formulations pédiatriques adaptées aux conditions des patients. En moins de 13 ans, sept nouveaux traitements ont été mis sur le marché grâce à des partenariats innovants. Le premier traitement lancé en 2007 est l'antipaludique ASAP (Coarsucam ${ }^{\circledR}$ ) - une association d'artémisinine $e^{5}[1](\rightarrow)$ et d'amodiaquine ${ }^{6}$ - développé avec un réseau de partenaires académiques (les universités de $(\rightarrow)$ Voir l'article Nobel de D. Mazier et $M$. Thellier, $m / s n^{\circ} 1$, janvier 2016, page 106 Bordeaux, d'Oxford, de Mahidol en Thaïlande, l'université Sains Malaysia, le Centre National de Recherche et de Formation sur le Paludisme au Burkina Faso, l'Institut Farmanguinhos/Fiocruz au Brésil) et produit par Sanofi, au Maroc. Les avantages clés de ce traitement sont représentés par son prix bas (moins de 1 \$ pour le traitement de l'adulte, 0,5\$ pour le traitement de

\footnotetext{
${ }^{3}$ Maladie du sommeil ou trypanosomiase africaine due à Trypanosoma brucei.

${ }^{4}$ La maladie de Chagas est provoquée par Trypanosoma cruzi.

${ }^{5}$ L'artémisinine est la substance active médicamenteuse isolée de la plante Artemisia annua. Sa découverte a conduit Tu Youyou à recevoir le prix Nobel de physiologie ou médecine en 2015.

${ }^{6} 4$-aminoquinoline apparentée à la chloroquine.
} 
l'enfant), sa facilité d'utilisation (une prise par jour pendant 3 jours) et le fait qu'il ne soit pas breveté, ce qui facilite sa production par d'autres laboratoires de génériques. Depuis son lancement, plus de 430 millions de traitements ont été distribués dans 35 pays, essentiellement en Afrique. Une réussite tant dans le domaine de la santé publique que du partenariat public/privé soutenu financièrement par des donateurs, dont la France.

\section{L'innovation pour les patients négligés et à un prix abordable}

Le mandat de DNDi a toujours été de contribuer à un débat pour un changement de modèle en ce qui concerne la R\&D, notamment sur la question essentielle du coût. L'objectif est de dissocier ce que coûte la R\&D du prix final du médicament. Dans le domaine des maladies infectieuses, DNDi estime le coût d'une amélioration thérapeutique entre 10 et 40 millions d'euros et le coût de développement d'une nouvelle entité chimique (NEC) entre 100 et 150 millions d'euros en tenant compte du taux d'attrition ${ }^{7}$. Le cas concret du composé de la classe des oxaboroles ${ }^{8}$ SCyX-7158, une nouvelle molécule issue du programme d'optimisation de DNDi avec la société biotech californienne Anacor, pourrait devenir le premier traitement oral à dose unique contre la maladie du sommeil. Son coût réel de développement est estimé à 60 millions d'euros. Le développement de NEC fait partie de la stratégie R\&D à long terme de DNDi, afin d'apporter des médicaments qui révolutionnent le traitement des malades atteints de maladies négligées. Le portefeuille de recherche de l'organisation compte à ce jour $15 \mathrm{NEC}$, qui représentent un espoir pour les patients atteints de la maladie du sommeil, de la leishmaniose, de la maladie de Chagas, des filarioses ${ }^{9}$, du mycétome ${ }^{10}$ ou d'hépatite $C$.

Comment expliquer les coûts raisonnables de R\&D présentés ci-dessus, alors que les mêmes techniques de recherche que les entreprises pharmaceutiques sont utilisées ? Une comparaison directe entre les coûts de l'industrie et ceux des partenariats de développement de produits (PDP) - notamment parce que les contributions en nature des compagnies pharmaceutiques aux PDP ne sont pas prises en compte - n'est pas envisageable. Toutefois, DNDi et d'autres PDP offrent un modèle alternatif basé sur le mode collaboratif, sur celui de la non-profitabilité et sur une gestion différente de la propriété intellectuelle (produits en grande partie non brevetés). En effet, développer un médicament contre la maladie du sommeil n'attire pas les mêmes espoirs de rentabilité qu'un hypotenseur de dernière génération.

Ce modèle de R\&D en train de faire ses preuves pour les maladies tropicales ne pourrait-il offrir de nouveaux espoirs

\footnotetext{
${ }^{7}$ Indicateur permettant de mesurer le pourcentage de perte sur une période donnée.

${ }^{8}$ Composé formé de bore associé à des composants organiques.

${ }^{9}$ Maladie parasitaire due à une filaire, un ver nématode.

${ }^{10}$ Pseudotumeur inflammatoire localisée dans le tissu sous-cutané, contenant des grains actinomycosiques ou fongiques.
}

pour tous les patients négligés? DNDi et ses partenaires ont décidé de se confronter à deux nouveaux défis. Tout d'abord, I'hépatite $C$ : en effet, malgré un portefeuille de recherche abondant, les patients se retrouvent exclus du système à cause des coûts pharaoniques des médicaments. Aucune approche de santé publique n'est actuellement possible, alors que cette maladie est curable avec une nouvelle génération de médicaments et pourrait donc être éliminée. L'ambition de DNDi, avec l'aide du partenaire industriel égyptien Pharco, est de développer un traitement à un prix abordable, moins de 300 dollars, pan-génotypique et accessible au plus grand nombre de patients. La seconde initiative récemment lancée conjointement par DNDi et I'OMS, le Partenariat global pour une R\&D sur les antibiotiques ${ }^{11}$, s'adresse à un défi qui peut toucher chacun d'entre nous: l'antibiorésistance.

Il est clair que le modèle de DNDi n'est pas l'unique alternative à un changement systémique de la R\&D, mais nous voulons croire que l'approche de santé publique qui a été notre moteur depuis le début fournira des modèles et des résultats reproductibles par d'autres. Nous nous situons résolument dans le champ de l'expérimentation sociale : on identifie un problème, on teste des solutions, on les transforme en biens communs. En cette période de fortes controverses, c'est le moment ou jamais de s'engager en faveur des populations fragiles et négligées pour leur apporter des solutions viables et pérennes, et tout simplement le droit de se soigner [2]. $\diamond$ An alternative and innovative Research and Development model to ensure access to medicines - Neglected diseases, neglected populations, and biomedical innovation system

\section{LIENS D’INTÉRÊT}

L'auteur déclare n'avoir aucun lien d'intérêt concernant les données publiées dans cet article.

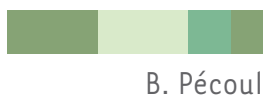

Drugs for Neglected Diseases initiative

(DNDi, initiative Médicaments contre les Maladies Négligées)

Chemin Louis-Dunant 15

1202 Genève, Suisse.

bpecoul@dndi.org

\section{RÉFÉRENCES}

1. Mazier D, Thellier M. Prix Nobel de Médecine 2015 (William C. Campbell, Satoshi Omura et Youyou Tu). Youyou Tu, de Mao Zedong au Prix Nobel. Med Sci (Paris) 2016; 32 : 106-9.

2. Maxmen A. Busting the billion-dollar myth: how to slash the cost of drug development : a non-profit organization is proving that new drugs don't have to cost a fortune. Can its model work more broadly? Nature 2016 ; $536: 388-90$.

${ }^{11}$ Global Antibiotic Research and Development Partnership (GARDP) : http://www.dndi.org/ diseases-projects/gardp/

TIRÉS À PART

B. Pécoul 